\title{
A Definitive Diagnosis of Mucosa-associated Lymphoid Tissue Lymphoma Made at a Second Biopsy
}

\author{
Tsutomu Igarashi', Akira Shimizu², Hiroki Yamaguchi', \\ Yoshimitsu Fukushima ${ }^{4}$, Toru Igarashi ${ }^{5}$ and Hiroshi Takahashi ${ }^{1}$ \\ ${ }^{1}$ Department of Ophthalmology, Nippon Medical School \\ ${ }^{2}$ Department of Pathology (Analytic Human Pathology), Nippon Medical School \\ ${ }^{3}$ Department of Hematology, Nippon Medical School \\ ${ }^{4}$ Department of Radiology, Nippon Medical School \\ ${ }^{5}$ Department of Pediatrics, Nippon Medical School
}

\begin{abstract}
Introduction: A case of mucosa-associated lymphoid tissue (MALT) lymphoma, the most frequent of the various conjunctival lymphoproliferative disorders, in which the initial biopsy was inconclusive but the second biopsy provided a definitive diagnosis, is reported.

Case Report: A 26-year-old woman with a 3-month history of bilateral conjunctival swelling was referred by a local physician for suspected MALT lymphoma. A salmon-pink elastic swelling was found to involve both eyes and to extend from the lower palpebral conjunctiva to the bulbar conjunctiva. Tonsillar swelling was also found, and ophthalmologic (left eye) and otolaryngologic biopsies were therefore performed simultaneously under general anesthesia. The otolaryngologic diagnosis was chronic tonsillitis. Light microscope examination of the conjunctival tissue showed proliferation of lymphocytes and small aggregates of small to medium-sized atypical lymphocytes. On immunohistochemical studies, atypical lymphocytes were positive for CD20 and CD79a, but differentiation to plasmacytes was not prominent, and neither Dutcher bodies nor evidence of immunoglobulin light chain restriction was found. The results were not incompatible with MALT lymphoma but were not definitive. A second biopsy of the right eye was therefore performed 3 months later. Staining with hematoxylin and eosin showed proliferation of small lymphocytes and monocytoid B cells and differentiation to plasmacytes. The hyperplastic cells were positive for CD19, CD79a, and CD20, and their cytoplasm were positive for Bcl-2 and slightly positive for Bcl-6. Cells positive for CD38 were noted where differentiation to plasmacytes and immunoglobulin light chain $\kappa$ restriction was evident on immunohistochemical studies and in situ hybridization. The Ki-67-positivity rate was approximately 5\%. The results of paraffin-embedded tissue section fluorescence in situ hybridization were negative for MALT-1 (18q21). A diagnosis of MALT lymphoma was made, and treatment with rituximab was started.

Discussion: Few findings lead directly to a definitive diagnosis of MALT lymphoma, and its differential diagnosis from benign lymphoproliferative disorders is difficult. In the present case definitive diagnosis was possible only after a second biopsy. This case suggests repeated biopsy may be necessary when a single biopsy is not definitive.

(J Nippon Med Sch 2013; 80: 475-480)
\end{abstract}

Key words: conjunctiva, definitive diagnosis, mucosa-associated lymphoid tissue, mucosaassociated lymphoid tissue lymphoma, second biopsy

Correspondence to Tsutomu Igarashi, MD, PhD, Department of Ophthalmology, Nippon Medical School, 1-1-5

Sendagi, Bunkyo-ku, Tokyo 113-8602, Japan

E-mail: tutomu@nms.ac.jp

Journal Website (http://www.nms.ac.jp/jnms/) 


\section{Introduction}

Mucosa-associated lymphoid tissue (MALT) occurs in mucosal tissue invaded by antigens and is a lymphatic apparatus peculiar to mucous membranes which governs local immunity. Immune cells that induce and control the activity of mucosal immunity systems aggregate in MALT, and antigen-specific lymphocytes thereby induce home via the common mucosal immune system on the effective tissues of the lamina propria mucosa and glandular tissues and produce secretions consisting chiefly of immunoglobulin A. The conjunctiva contains conjunctiva-associated lymphoid tissue, a type of MALT. The term "MALT lymphoma" was first applied in 1983 by Isaacson et al. ${ }^{1}$ to a malignant lymphoma developing from MALT, and similar lesions have since been found at various locations in the gastrointestinal tract, lung, bronchi, thyroid gland, and other systems ${ }^{2}$. In the field of ophthalmology, although MALT lymphoma originating in the orbit or conjunctiva has lower malignant potential and metastasizes less frequently than usual malignant lymphomas, MALT lymphoma must be clearly differentiated from systemic malignant lymphoma. ${ }^{3}$ For diagnosis and treatment in the present case, conjunctival tumors were excised from both eyes and were subjected to pathological and immunohistochemical analysis. We describe the details of this case, which was diagnosed as MALT conjunctival malignant lymphoma originating in the palpebral conjunctiva of both eyes.

\section{Case Report}

A 26-year-old woman with a 3-month history of bilateral conjunctival swelling and suspected MALT lymphoma was referred to the Nippon Medical School Musashikosugi Hospital, where she underwent hematologic, otorhinolaryngologic, and ophthalmologic examinations. Salmon-pink elastic conjunctival swelling, extending from the lower palpebral conjunctiva to the bulbar conjunctiva (Fig. la and b), was found in both eyes. No other ophthalmologic abnormality was found.

${ }^{18} \mathrm{~F}$-fluorodeoxyglucose (FDG) positron emission tomography (PET)/computed tomography (CT) was performed with a PET-CT scanner and a 16-slice multislice CT scanner (Gemini TF 16, PhilipsHealthcare, Amsterdam, Netherlands). Early (Fig. 2a and b) and delayed (Fig. 2c and d) images were acquired 60 and 120 minutes after administration of FDG. The PET/CT images showed intense FDG uptake in the bilateral tumors located in the lower palpebral conjunctiva and bulbar conjunctiva (Fig. 2 e and f) (maximum standardized uptake value $\left[\mathrm{SUV}_{\max }\right]$ right side early: 2.12; right side delayed: 2.55; left side early: 1.81; right side delayed: 2.18). Right-sided palatine tonsillar swelling with high FDG uptake was seen $\left(\mathrm{SUV}_{\max }\right.$ early: 4.50; delayed: 5.18).
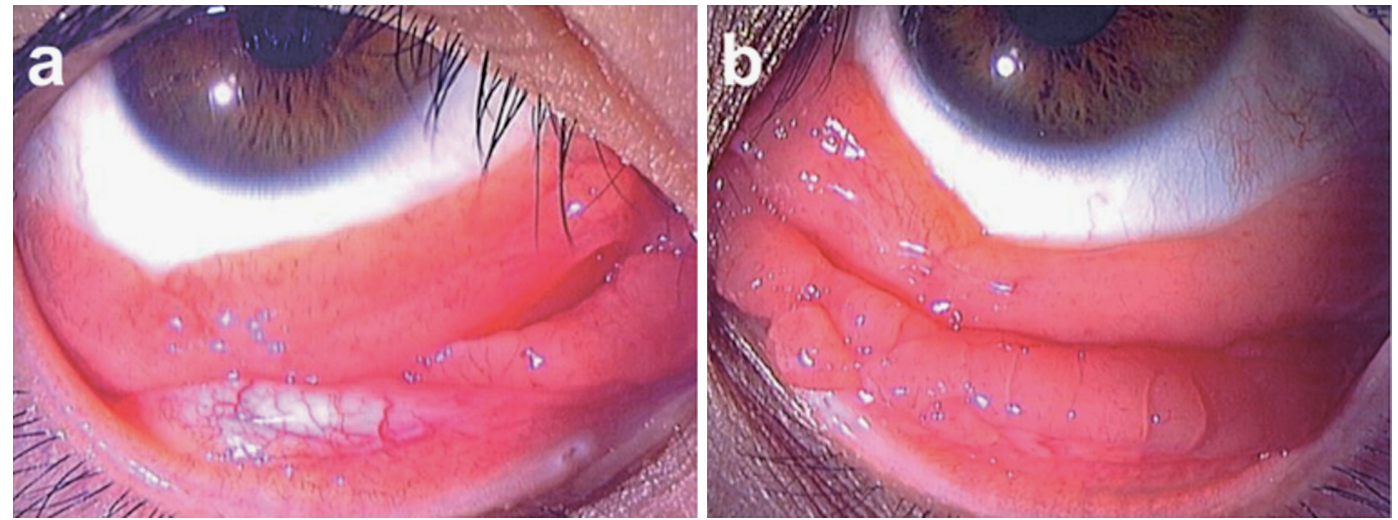

Fig. 1 Slit-lamp examination at presentation. Slit-lamp examination reveals a salmon-pink conjunctival tumor (a, right eye; b, left eye) 
a

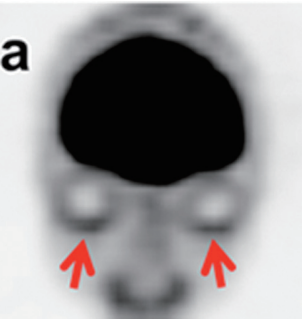

Early image
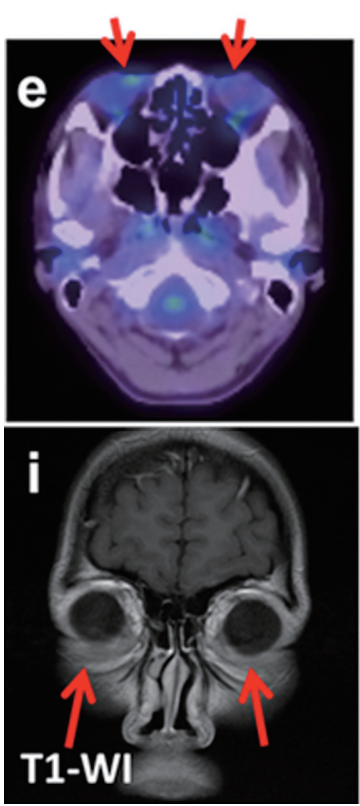
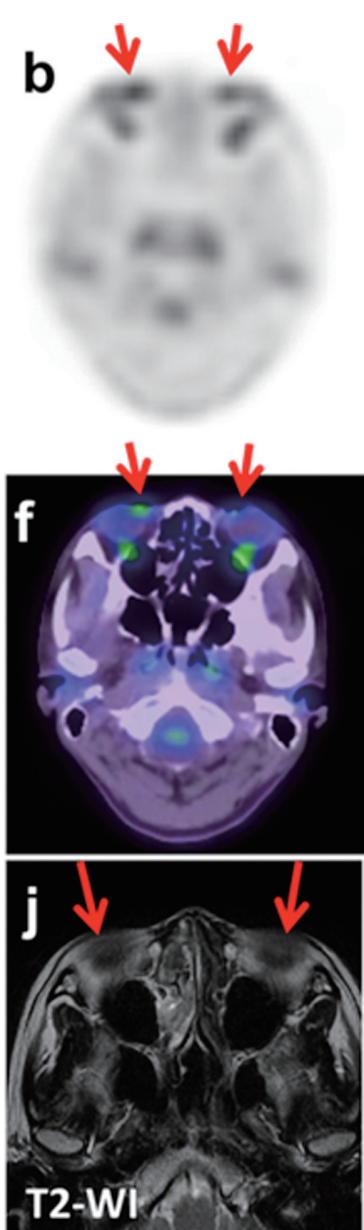
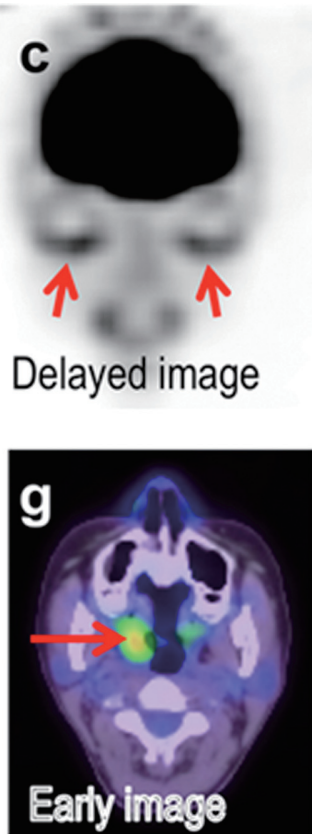
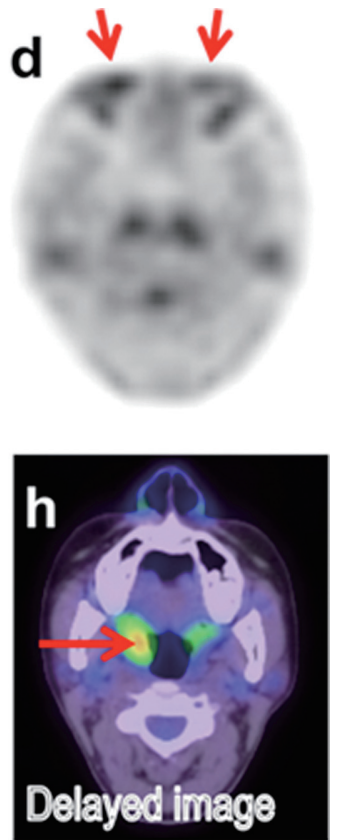

Fig. 2 18F-FDG-PET/CT and magnetic resonance images of the present case.

Early images of PET/CT show intense FDG uptake in the tumors in lower palpebral conjunctiva and bulbar conjunctiva of both eyes (SUV $\max$ right side: 2.12; left side: 1.81) (a, b, e). Delayed images of PET/CT show retention of uptake in the lesions $\left(\mathrm{SUV}_{\max }\right.$ right side: 2.55; left side: 2.18) (c, d, f). Right-sided palatine tonsillar swelling with high ${ }^{18} \mathrm{~F}-\mathrm{FDG}$ uptake is seen ( $\mathrm{SUV}_{\max }$ early: 4.50; delayed: 5.18). Determining whether this lesion was chronic tonsillitis or MALT was difficult with only PET/CT images (g, h). With magnetic resonance, T1weighted images show low intensity (i), T2-weighted images show high intensity (j), and T1-weighted images show contrast enhancement in the lesions.

Determining whether this lesion was chronic tonsillitis or MALT was difficult with only PET/CT images (Fig. $\mathbf{2 g}$ and $\mathbf{h}$ ). Magnetic resonance imaging was also performed. The T1-weighted images showed low intensity (Fig. 2i), and T2-weighted images showed high intensity (Fig. 2j), with contrast enhancement in the tumors. These findings did not contradict the diagnosis of MALT.

Because tonsillar swelling was also found, ophthalmologic (left eye) and otolaryngologic biopsies were performed simultaneously under general anesthesia. The tonsillar swelling was diagnosed pathologically as chronic tonsillitis.

Pathological examination of the conjunctival tissue from the left eye (Fig. 3) showed proliferation of small to medium-sized atypical lymphocytes. Atypical lymphocytes were positive for $\mathrm{CD} 20$ and CD79a, but differentiation to plasmacytes was not prominent, and neither Dutcher bodies nor evidence of immunoglobulin light chain restriction was found.

The results were not incompatible with MALT lymphoma but were not definitive, and a second biopsy of the right eye was therefore performed 3 months later. Pathological examination of conjunctival tissue from the right eye (Fig. 4) showed proliferation of small lymphocytes and monocytoid B cells with large irregular lymph follicles. In addition, a few scattered transformed centroblast-like cells were present. In this case, however, centrocyte-like cells were not prominent in

J Nippon Med Sch 2013; 80 (6) 

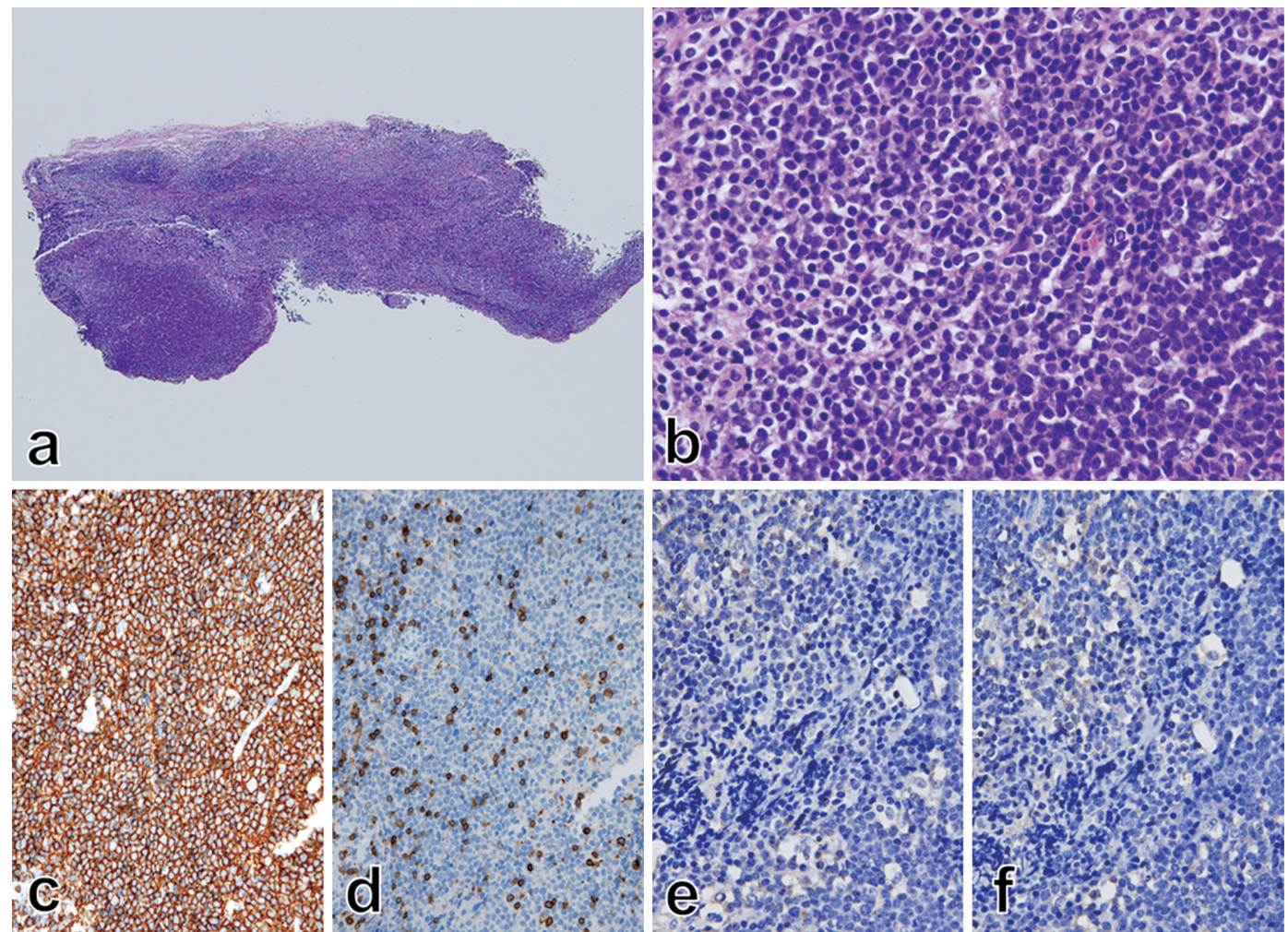

Fig. 3 Pathological findings of conjunctival tissue of the left eye (first biopsy).

(a) Low magnification $(\times 40)$ of conjunctival tissue shows monotonous proliferation of lymphocytes without the formation of lymph follicles (hematoxylin and eosin [HE] stain). (b) Aggregated lymphocytes are small to medium-sized lymphocytes with relatively abundant pale cytoplasm, indicating a monocytoid appearance (HE stain, $\times 600$ ). Plasma cell differentiation and follicular colonization are not prominent, and Dutcher bodies were not detected. Atypical lymphocytes are positive for $\mathrm{CD} 20(\mathrm{c}, \times 400)$ and negative for $\mathrm{CD} 3(\mathrm{~d}, \times 400)$. On in situ hybridization for $\kappa(\mathrm{e}, \times 600)$ and $\lambda$ (f, $\times 600$ ), immunoglobulin light chain restriction is not evident, because plasmacytic differentiation is not prominent in the specimen.

the conjunctival tissue. Cells positive for CD38 were prominent in regions with differentiation to plasmacytes; in parts of these regions immunohistochemical studies and in situ hybridization showed that $\kappa$ was greater than $\lambda$ by a factor of 10 or more, indicating immunoglobulin light chain $\mathrm{\kappa}$ restriction (Fig. 5).

Atypical lymphocytes were positive for CD19, $\mathrm{CD} 79 \mathrm{a}$, and $\mathrm{CD} 20$, and their cytoplasm was positive for Bcl-2 and slightly positive for Bcl-6. The Ki-67positivity rate was approximately $5 \%$. A small number of CD3s was also observed, and CD5 and CD43 were found in a similar distribution. Other findings were CyclineD- and CD10- The results of paraffin-embedded tissue section fluorescence in situ hybridization were negative for MALT-1 (18q21). A diagnosis of MALT lymphoma was made, and treatment with rituximab was started.

\section{Discussion}

Lymphoid neoplasms originating in the orbit and subconjunctiva are broadly classified as malignant lymphoma or benign reactive lymphoid hyperplasia, which includes inflammation and pseudolymphoma. Chronic inflammation is clearly associated with an increased risk of malignancy ${ }^{4}$. The most frequent malignant lymphoma is MALT lymphoma $a^{5}$. Despite their low malignant potential, MALT lymphomas differ little from reactive lymphoid hyperplasia in age at onset, clinical presentation, and morphological findings, and differential diagnosis is difficult. Both disorders, as in the present case, characteristically present with well-defined, smooth-surfaced, salmonpink lesions and are slowly progressive. Histopathological and immunohistochemical 

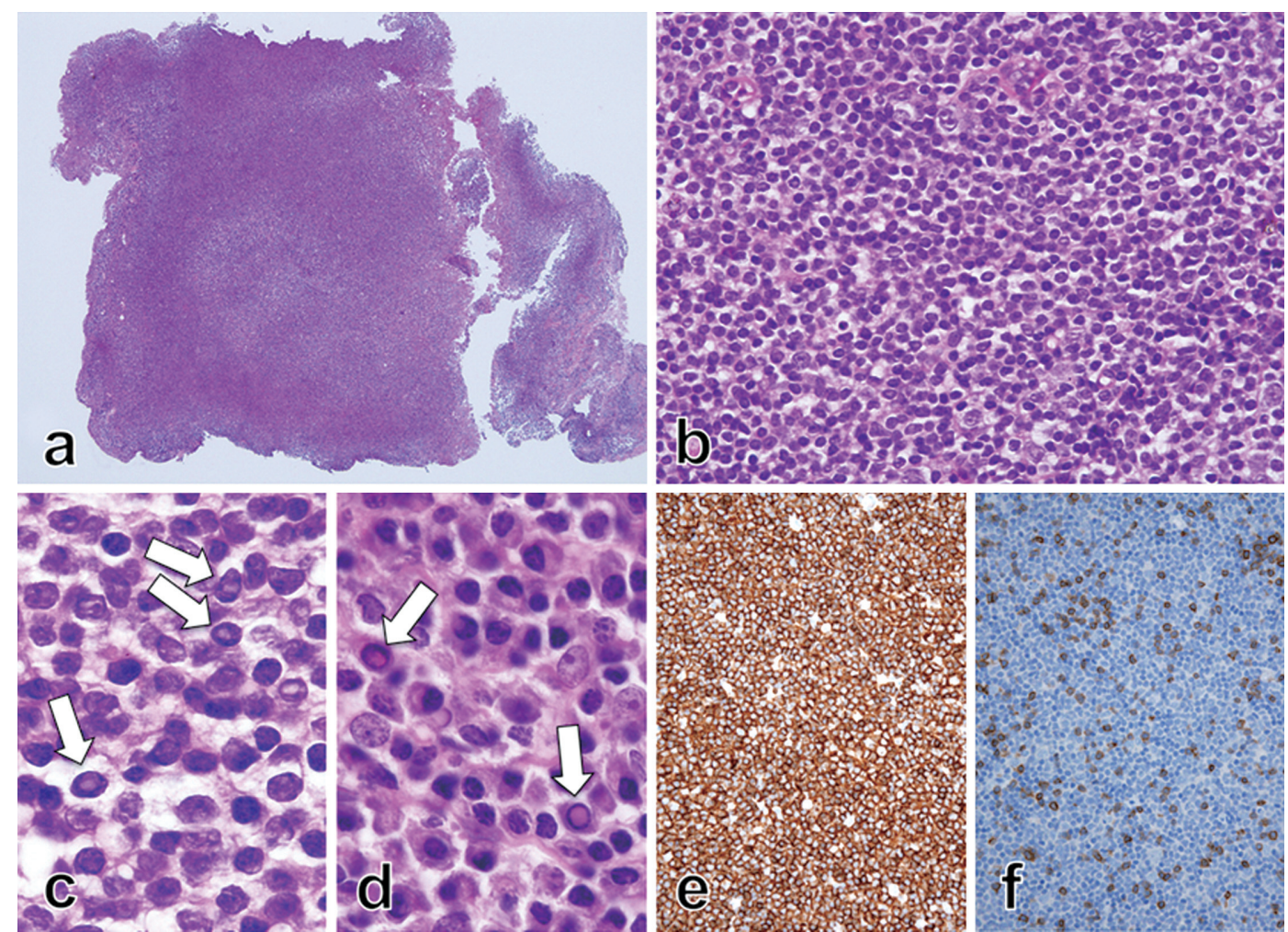

Fig. 4 Pathological findings of conjunctival tissue of the right eye (second biopsy).

(a) Low magnification $(\times 40)$ of conjunctival tissue shows monotonous proliferation of lymphocytes with irregular large lymph follicles (HE stain, $\times 600$ ). (b) Aggregated lymphocytes are small to medium-size lymphocytes with relatively abundant pale cytoplasm, indicating a monocytoid appearance (HE stain, $\times 600$ ). (c) Dutcher bodies (arrows in c and d) are prominent in monocytoid B cells (c: HE stain, $\times 1,000$ ) and plasmacytic cells (d: HE stain, $\times 1,000)$. Atypical lymphocytes are positive for CD20 (e, $\times 400)$ and negative for CD3 (f, $\times 400)$
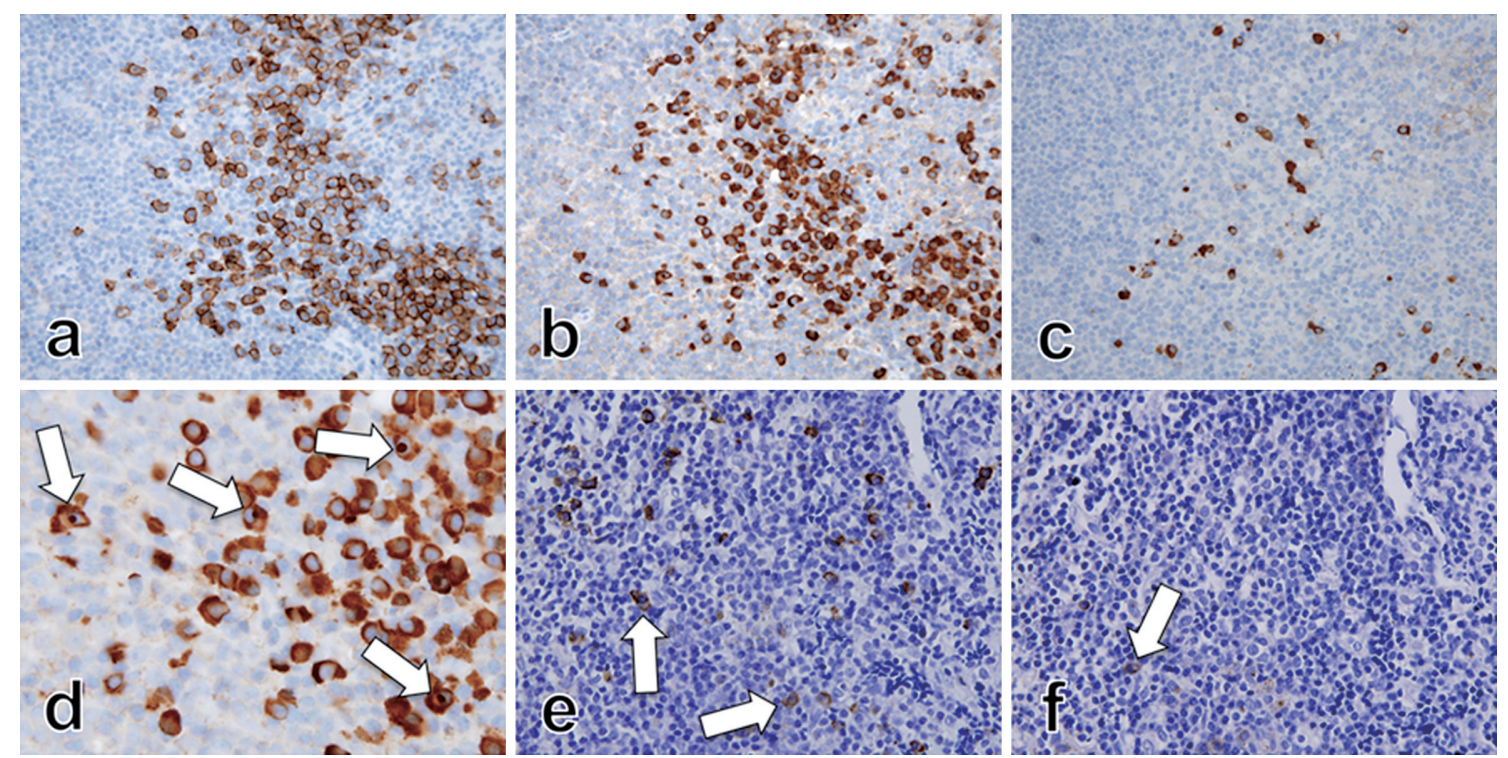

Fig. 5 Plasmacytic differentiation and immunoglobulin light chain restriction (second biopsy).

Immunohistochemical studies for $\operatorname{CD} 38(\mathrm{a}, \times 600)$ show prominent plasmacytic differentiation. Immunohistochemical studies for $\kappa(b, \times 600)$ and $\lambda(c, \times 600)$ show immunoglobulin light chain $\kappa$ restriction. In addition, immunoglobulin light chain $\kappa$ is detected in Dutcher bodies (arrows in $\mathrm{d}$ : $\times 1,000$ ). In situ hybridization for $\kappa(\mathrm{e}: \times 600)$ and $\lambda(\mathrm{f}: \times 600)$ also shows immunoglobulin light chain $\kappa$ restriction. 
examinations and gene searches are useful for differentiating MALT lymphoma from benign reactive lymphoid hyperplasia. Because reactive lymphoid hyperplasia is not a neoplastic proliferation, it exhibits cellular polymorphism on histopathological examination and comprises lymphocytes that exhibit T-cell-predominant polyclonal proliferation. MALT lymphoma, in contrast, comprises small to medium-sized lymphoid cells, and in the conjunctival epithelium, shows proliferation of small centrocyte-like cells and monocytoid B cells, with relatively little metaplasia, which casts doubt on monoclonality, together with an intermixture of cells with Dutcher bodies having intranuclear inclusions and plasmacytoid cells ${ }^{1}$. Immunohistochemical studies are positive for anti-Bcell monoclonal antibodies, negative for anti-T-cell monoclonal antibodies, and positive for immunoglobulin $\kappa$ - or $\lambda$-chain for classification of the light chain restriction. When more detailed investigation is required, a molecular biological search is performed, immunoglobulin gene rearrangement is confirmed, and $B$ cell monoclonality is determined ${ }^{3}$.

The standard treatments for conjunctival lymphoma includes radiotherapy, surgical excision, and systemic single-agent or combination chemotherapy $^{6}$. Recently, tumor regression in patients with conjunctival MALT lymphoma has been treated with antibiotics ${ }^{7}$, topical chemotherapy ${ }^{8}$, intralesional interferon ${ }^{9}$, and intravenous rituximab ${ }^{10}$. Rituximab is a recombinant chimeric anti-CD20 monoclonal antibody used as first-line and salvage therapy for a wide variety of B-cell non-Hodgkin lymphomas ${ }^{11}$. The therapeutic effect of rituximab is the result of antibody-dependent cell- and complement-mediated cytotoxicity ${ }^{12}$. The use of conventional doses of intravenous rituximab in ocular MALT lymphoma has been only anecdotally investigated. The largest report suggests modest and transient benefit, with some cases of durable remission in conjunctival MALT lymphoma ${ }^{10}$. In the present case, rituximab monotherapy was performed, and no recurrence was found over the following month.
In conclusion, a rigorous approach to the initial diagnosis and staging of MALT lymphoma is needed. The recognition of MALT lymphoma requires appropriate immunohistochemical staining or genetic confirmation or both.

Conflict of Interest: The authors declare that they have no conflict of interest.

\section{References}

1. Isaacson $\mathrm{P}$, Wright $\mathrm{DH}$, Jones DB: Malignant lymphoma of true histiocytic (monocyte) macrophage) origin. Cancer 1983; 51: 80-91.

2. Kawashima $T$, Nishimura $H$, Akiyama $H$, et al: Primary pulmonary mucosa-associated lymphoid tissue lymphoma combined with idiopathic thrombocytopenic purpura and amyloidoma in the lung. J Nippon Med Sch 2005; 72: 370-374.

3. Sjo LD: Ophthalmic lymphoma: epidemiology and pathogenesis. Acta Ophthalmol 2009; 87: 1-20.

4. Tanno T, Matsui W: Development and Maintenance of Cancer Stem Cells under Chronic Inflammation. J Nippon Med Sch 2011; 78: 138-145.

5. Cho EY, Han JJ, Ree HJ, et al:: Clinicopathologic analysis of ocular adnexal lymphomas: extranodal marginal zone b-cell lymphoma constitutes the vast majority of ocular lymphomas among Koreans and affects younger patients. Am J Hematol 2003; 73: 8796

6. Ferreri AJ, Assanelli A, Crocchiolo R, et al: Therapeutic management of ocular adnexal MALT lymphoma. Expert Opin Pharmacother 2007; 8: 10731083.

7. Ferreri AJ, Ponzoni M, Guidoboni M, et al. Regression of ocular adnexal lymphoma after Chlamydia psittaci-eradicating antibiotic therapy. J Clin Oncol 2005; 23: 5067-5073.

8. Yu CS, Chiu SI, Ng CS, Chan HH, Tse RK: Localized conjunctival mucosa-associated lymphoid tissue (MALT) lymphoma is amenable to loca chemotherapy. Int Ophthalmol 2008; 28: 51-54.

9. Blasi MA, Gherlinzoni F, Calvisi G, et al.: Local chemotherapy with interferon-alpha for conjunctiva mucosa-associated lymphoid tissue lymphoma: a preliminary report. Ophthalmology 2001; 108: 559562.

10. Ferreri AJ, Ponzoni M, Martinelli G, et al.: Rituximab in patients with mucosal-associated lymphoid tissuetype lymphoma of the ocular adnexa. Haematologica 2005; 90: 1578-1579.

11. Cvetkovic RS, Perry CM: Rituximab: a review of its use in non-Hodgkin's lymphoma and chronic lymphocytic leukaemia. Drugs 2006; 66: 791-820.

12. Shan D, Ledbetter JA, Press OW: Apoptosis of malignant human B cells by ligation of CD20 with monoclonal antibodies. Blood 1998; 91: 1644-1652.

(Received, September 12, 2013)

(Accepted, October 11, 2013) 